\title{
Nefrocalcinosis como complicación de hipercalcemia por ingesta excesiva de calcio en el embarazo
}

\author{
Elizabeth Bedoya C. ${ }^{1}$, Natalia María Mazo C. ${ }^{2}$, Andrea Restrepo C. ${ }^{3}$, María Paula Suárez \\ G. ${ }^{4}$, Alejandro Giraldo Cardona. 5
}

${ }^{1}$ Médica General, Universidad Pontificia Bolivariana, Corporación IPS Comfamiliar Camacol Coodan, Medellín. ${ }^{2}$ Médica General, Universidad Pontificia Bolivariana, Clínica Universitaria Bolivariana, Medellín. ${ }^{3}$ Médica General, Universidad Pontificia Bolivariana, Editora médica, Fondo Editorial Corporación para Investigaciones Biológicas (CIB). Medellín. ${ }^{4}$ Médica General, Universidad Pontificia Bolivariana, IDIME Nueva Eps., Armenia. ${ }^{5}$ Gineco-Obstetra, Universidad CES, Servicio Gineco-Obstetricia, Clínica Saludcoop, Juan Luis Londoño de la Cuesta, Medellín. Colombia.

\section{RESUMEN}

Antecedentes: La hipercalcemia durante el embarazo es un hallazgo raro. La exposición prolongada a los altos niveles de calcio puede llevar a calcificaciones en distintos órganos maternos y en el feto puede llevar a abortos espontáneos y mortinatos. Caso clínico: Paciente primípara, con gestación de 20+4 semanas, remitida por falla renal aguda e hipertensión arterial. Ella reportó ingesta excesiva de queso, leche y multivitamínicos. Con calcio de 9,2 mg/dL y relación BUN/Creatinina de 6,8 se diagnostica intoxicación alimentaria y nefrocalcinosis medular por alta ingesta de calcio. Al cuarto día de hospitalización presenta sangrado vaginal y debido a las condiciones de la madre se termina el embarazo. Posteriormente presenta una mejoría clínica significativa y se da de alta. Discusión: La relevancia de este caso es que la presencia de hipercalcemia en el embarazo y su no identificación, aumenta el riesgo de aborto, y de hipocalcemia neonatal, que a su vez puede causar muerte materna. En este cuadro se pueden presentar varias complicaciones, siendo una de las más frecuentes la nefrocalcinosis, identificada en este reporte. Conclusión: La ingesta de calcio excesiva en gestantes puede causar nefrocalcinosis. Es importante la educación para evitar ésta y otras complicaciones.

\section{PALABRAS CLAVE: Hipercalcemia, embarazo, nefrocalcinosis, calcio, nefrolitiasis, leche}

\section{SUMMARY}

Background: Hypercalcemia during pregnancy is a strange finding. The long exposure to high calcium levels can take to calcifications in different maternal organs and it can lead to miscarriage and stillborn. Case presentation: A woman with 20+4 weeks' gestation, who was remitted because of an acute renal failure and hypertension. She reported excessive intake of cheese, milk and multivitaminics. With a calcium level of $9.2 \mathrm{mg} / \mathrm{dL}$ and BUN/Creatinine of 6.8 , diagnosed with food poisoning and medullary nephrocalcinosis as a result of the high calcium intake. At the fourth day at the hospital she presented vaginal bleeding and due to the conditions of the mother the pregnancy was ended. Afterwards she presented a significant clinical improvement and she was discharged from the hospital. Discussion: The relevance of this case is that the presence of hypercalcemia during pregnancy and not identifying it elevates the risk of miscarriage and neonatal hypocalcemia, which can cause maternal death. In this aspect, many complications can develop, 
being one of the most important the nephrocalcinosis, identified in this report. Conclusion: The excessive calcium intake in pregnant women can cause nephrocalcinosis. It is important to educate them to avoid this and other complications.

\section{KEY WORDS: Hypercalcemia, pregnancy, nephrocalcinosis, calcium, nephrolithiasis, milk}

\section{INTRODUCCIÓN}

La hipercalcemia durante el embarazo es un hallazgo raro, en la mayoría de los casos se da principalmente por hiperparatiroidismo primario y aún, esta entidad se presenta con poca frecuencia durante la gestación $(1,2)$. Su incidencia exacta es desconocida, pero en los casos publicados en la literatura mundial se estima alrededor de 150 casos (3).

Debido a que los requerimientos de calcio durante el embarazo varían según el área geográfica, determinar un valor normal estándar a nivel mundial es complicado. El valor a partir del cual comienzan a hacerse manifiestos los efectos adversos es muy variable, pero en general se ha definido exceso de calcio como un nivel diario de al menos 4-5 gramos. Esto se ha determinado mediante estudios realizados en mujeres en embarazo que consumen altas cantidades de suplemento de calcio y que ingieren altas cantidades de productos lácteos, como es el caso de la paciente que se presentará posteriormente $(4,5,6)$.

La exposición prolongada a altos niveles de calcio puede llevar a calcificaciones en distintos órganos, siendo los más comunes la córnea y los riñones. En estos casos el feto también puede verse afectado, alterando su sistema circulatorio, suprimiendo su función paratiroidea y provocando hipocalcemia neonatal; asociada también a abortos espontáneos y mortinatos $(2,6,7)$.

El objetivo de esta comunicación es presentar un caso de nefrocalcinosis secundario a hipercalcemia por ingesta excesiva de lácteos y calcio durante el embarazo.

\section{Caso clínico}

Mujer de 30 años, raza blanca. Con antecedentes quirúrgicos de laparoscopia por miomatosis. Refiere consumo de ácido fólico y polivitamínico del embarazo 1 o 2 comprimidos/día (160 mg de carbonato de calcio $+400 \mathrm{U}$ de vitamina D3). Cursa su primer embarazo con edad gestacional de 20 semanas+4 por ecografía obstétrica del primer trimestre. Fecha de última menstruación desconocida.

Consulta inicialmente en urgencias en un Hospital del municipio de Apartadó (Antioquia, Colombia.) por cuadro de 3 días de evolución de dolor en hipogastrio e hipocondrio derecho sin irradiación, con 2 episodios de heces blandas, sin moco ni sangre, vómito en repetidas ocasiones de contenido bilioso y alimentario en algunas ocasiones. Refirió 2 días de anorexia por mala tolerancia a la vía oral, además de fiebre subjetiva y disminución de la cantidad y frecuencia miccional desde el día anterior. No acusa sangrado genital. Es remitida desde Apartadó hacia Medellín (Antioquia, Colombia), a una Institución Prestadora de Servicios de Salud, con sospecha de falla renal aguda, síndrome hipertensivo asociado al embarazo, amenaza de aborto y miomatosis uterina. La paciente ingresa al servicio de salud en Medellín sin medicación alguna y presenta los exámenes que le realizaron en Apartadó. Trae ecografía abdominal que reporta nefrocalcinosis medular bilateral, mioma uterino de $94 \times 83 \mathrm{~mm}$, sin patología biliar y un parcial de orina con eritrocitos de 30 x CAP (Campo de Alto Poder). Es evaluada, encontrándose afebril al tacto, con presión arterial de 156/99, frecuencia respiratoria de $18 / \mathrm{min}$, saturación de oxígeno $98 \%$, pulso $90 /$ min. El abdomen se encuentra grávido con altura uterina de $22 \mathrm{~cm}$, frecuencia cardiaca fetal de 136/ min, sin actividad uterina, leve dolor a la palpación en hipocondrio derecho, Murphy negativo. Extremidades sin edemas, pulsos periféricos presentes, y sin pérdidas vaginales, no se realiza tacto vaginal, no presenta déficit neurológico.

En día 1 de hospitalización, se realizan nuevos paraclínicos, encontrándose leucocitosis, trombocitopenia leve, calcemia 9,2 mg/dL, alteración de función renal y hepática. Debido a la zona geográfica de que proviene la paciente, el médico general a cargo, sospecha cuadro infeccioso tipo leptospirosis, por lo que solicita urocultivo, CK total e IgM para leptospira, además de gota gruesa, el cual fue descartado posteriormente, al obtener resultados negativos. El mismo día que ingresó al servicio de salud en Medellín es valorada por médico de la Unidad de Cuidados Intensivos quien descarta criterios para ser admitida en dicha unidad, descarta un posible HELLP, y refiere que hay datos que sugieren una insuficiencia renal crónica con su consecuencia hipertensiva: nefrocalcinosis y relación BUN/Creatinina 6,8 y además se sospecha una descompensación aguda por el embarazo. Inicia tratamiento de la hipertensión arterial con metoprolol $50 \mathrm{mg}$ vía oral cada 12 horas, dieta baja en sal 
y sin restricción hídrica, e inicia tratamiento antibiótico (ceftriaxona $2 \mathrm{~g}$ i.v./día + metronidazol 7,5 mg/ $\mathrm{kg}$ cada 6 horas). La hipertensión arterial comienza a controlarse con esos medicamentos.

En el día 2 de hospitalización, luego de realizarle los paraclínicos de control y otros nuevos, es valorada por médico internista quien diagnostica una falla renal mixta (prerrenal por la deshidratación y renal por nefrocalcinosis), intoxicación alimentaria y nefrocalcinosis medular por alta ingesta de calcio, ya que luego de indagar exhaustivamente en la anamnesis, se encontró que la paciente ingería grandes y repetidas cantidades de queso y leche, y además consumía el multivitamínico, por lo cual se suspende el calcio y el calcitriol (1-alpha,25-dihidroxicolecalciferol). Se indica una dieta baja en calcio, hidratación exhaustiva con solución salina al $0,9 \%$, y continuar con ceftriaxona + metronidazol por probable foco infeccioso a nivel gastrointestinal. Durante las valoraciones, se observaba hematuria en la bolsa recolectora de orina, concluyéndose que era secundaria a la nefrocalcinosis.

En el día 3 de hospitalización, se agrega alfametildopa de $500 \mathrm{mg}$ vo. c/12 horas, debido a la tendencia a aumentar las cifras tensionales. Proteinuria de $24 \mathrm{~h}$ de $1,2 \mathrm{~g}$. La ecografía de vías uri-

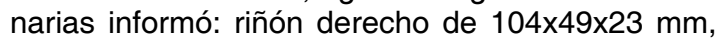
cortical $10 \mathrm{~mm}$; riñón izquierdo de 125×57×27 mm, cortical $10 \mathrm{~mm}$. Ambos riñones presentan aumento en la ecogenicidad de la corteza y disminución en la diferenciación corticomedular probablemente por nefropatía aguda, aumento leve del tamaño renal izquierdo. La ecografía obstétrica reporta embarazo de 21 semanas +4 días, peso fetal estimado 387 gramos, percentil 33, índice de líquido amniótico 14 y placenta grado I. Evaluada por nefrología, diagnostican proteinuria no nefrótica y posible falla renal crónica agudizada.

En el día 4 de hospitalización, la paciente presenta sangrado vaginal rojo rutilante sin coágulos. Al tacto vaginal se encuentra cérvix dilatado en 1 $\mathrm{cm}$, frecuencia cardiaca fetal positiva. El ginecoobstetra indica a la paciente reposo absoluto, reserva 2 unidades de glóbulos rojos, ciproato de hidroprogesterona $500 \mathrm{mg}$ intravenoso, nifedipino $30 \mathrm{mg}$ vía oral $\mathrm{c} / 12$ horas. Más tarde debido a la edad gestacional y al deterioro materno se considera la posibilidad de terminar el embarazo luego del consenso de varios especialistas. El edema continúa progresando hasta llegar a anasarca, presenta hiperreflexia, se identifica un deterioro materno rápido y progresivo, con empeoramiento de las funciones plaquetarias. Se explica a la paciente la importancia de terminar con el embarazo y el pobre pronóstico fetal, otorgando su consentimiento. Se inicia misoprostol intravaginal en dosis de $50 \mathrm{mi}$ crogramos.
En el día 5 de hospitalización y aproximadamente a las 9 horas, rompe membranas con líquido claro y expulsa feto muerto de sexo masculino, sin malformaciones aparentes al examen físico, con un peso de $300 \mathrm{~g}$, se realiza revisión uterina y curetaje obstétrico bajo anestesia epidural. Se indica oxitocina profiláctica y queda con sonda vesical para contabilizar diuresis.

En el día 6 de hospitalización, debido a que la paciente continuaba estable, no tuvo ninguna complicación y los nitrogenados comenzaron a descender sus valores, fue pasada de la Unidad de Cuidados Intensivos a hospitalización.

En el día 7 de hospitalización, el nefrólogo suspende la alfametildopa e indica metoprolol $50 \mathrm{mg}$ $\mathrm{c} / 12$ horas. Más tarde es valorada y dada de alta por su buena evolución y se indica continuar tratamiento por medicina interna y nefrología.

Dos días después (día 9), la paciente refiere mejoría y se suspenden el metronidazol y la ceftriaxona. Se inicia sulfato ferroso y se cambia el nifedipino por amlodipino $10 \mathrm{mg} \mathrm{c} / 12$ horas (día 10). En el día 11 es dada de alta por medicina interna y nefrología gracias a una mejoría clínica importante.

\section{DISCUSIÓN}

La hipercalcemia puede afectar tanto la madre como al feto, infrecuentemente las mujeres desarrollan una hipercalcemia grave presentando anorexia rápidamente progresiva, náuseas y vómitos, confundiéndose con hiperémesis gravídica. Estas pacientes deben ser hidratadas rápidamente para que la hipercalcemia no se agrave. Además hay que identificar otras entidades asociadas como la hipertensión arterial, el letargo, la confusión, el cansancio, la debilidad, el dolor abdominal y la pancreatitis aguda, la deshidratación, los cambios mentales, ya que hacen parte de este cuadro y con riesgo de muerte materna, por lo que requieren un tratamiento urgente y agresivo (5).

En la hipercalcemia se pueden presentar varias complicaciones, siendo las más frecuentes: la nefrocalcinosis (8), identificada en este reporte y la nefrolitiasis, enfermedades óseas, pancreatitis, infección del tracto urinario, abortos espontáneos y crisis hipercalcémicas, aunque la mayoría de las veces se trata de sintomatología inespecífica y son de intensidad moderada. Todo lo anterior justifica lo poco común del caso expuesto, en el cual la paciente, con sus antecedentes de consumo de multivitamínicos (5) y la alta ingesta de alimentos ricos en calcio, favoreció la presentación y la manifestación de los síntomas y signos descritos $(5,6)$.

Es evidente que este cuadro es de difícil diagnóstico, por lo que requiere que sea una entidad conocida (6), tanto por especialistas como por los 
médicos en general, para que tengan la destreza de identificar y reconocerla precozmente, y así realizar una intervención en el lugar y momento adecuado para evitar las complicaciones mencionadas anteriormente, que pueden agravarse y causar inclusive la muerte materna, aunque en la literatura aún no se han encontrado reportes de estos casos (9).

\section{CONCLUSIÓN}

Hay evidencia en la literatura que demuestra que la ingesta excesiva de calcio puede provocar consecuencias graves para la madre y el feto, incluida la nefrocalcinosis. Es necesario educar a las pacientes acerca de las consecuencias propias y fetales que podría traer el consumo excesivo de calcio, cualquiera sea su origen, para prevenir su alteración durante el embarazo.

AGRADECIMIENTOS: A Erika Tatiana Loaiza Echeverry por su asesoría metodológica.

\section{REFERENCIAS}

1. Prentice A. Maternal calcium requirements during pregnancy and lactation. Am J ClinNutr 1994;59(2 Suppl):477S-83S.
2. Picolos MK, Sims CR, Mastrobattista JM, Carroll MA, Lavis VR. Milk-alkali syndrome in pregnancy. Obstet Gynecol 2004;104(5 Pt 2):1201-4.

3. Abraham P, Ralston SH, Hewison M, Fraser WD, Bevan JS. Presentation of a PTHrP-secreting pancreatic neuroendocrine tumour, with hypercalcaemic crisis, pre-eclampsia, and renal failure. Postgrad Med $\mathrm{J}$ 2002;78(926):752-3

4. Kato Y, Sato K, Sata A, Omori K, Nakajima K, Tokinaga $\mathrm{K}$, et al. Hypercalcemia induced by excessive intake of calcium supplement, presenting similar findings of primary hyperparathyroidism. Endocr $\mathrm{J}$ 2004;51(6):557-62.

5. Felsenfeld AJ, Levine BS. Milk alkali syndrome and the dynamics of calcium homeostasis. Clin J Am SocNephrol 2006;1(4):641-54.

6. Medarov BI. Milk alkali syndrome. Mayo Clin Proc 2009;84(3):261-7.

7. Bergel E, Barros AJD. Effect of maternal calcium intake during pregnancy on children's blood pressure: a systematic review of the literature. BMC Pediatrics 2007;7(15):1-9.

8. Ennen CS, Magann EF. Milk-Alkali syndrome presenting as acute renal insufficiency during pregnancy. Obstet Gynecol 2006;108(3 Pt 2):785-6.

9. George S, Clark JDA. Milk alkali syndrome-an unusual syndrome causing an unusual complication. Postgrad Med J 2000; 76(897):422-3. 